\title{
Prosthodontic Rehabilitation of Maxillary Anterior Ridge Defect With Fixed - Removable Bridge System: A Case Report
}

\author{
Rasmi $\mathrm{M}^{1}$,Gilsa K Vasunni ${ }^{2}$, Mehul R Mahesh ${ }^{3}$,Bennett Atlin Correya ${ }^{3}$,Reshma \\ Premkumar ${ }^{4}$,Prasanth Manohar B ${ }^{5}$ \\ ${ }^{1}$ Junior Resident, Dept. of Prosthodontics, Govt. Dental College, Kozhikode \\ ${ }^{2}$ Principal, Govt. Dental College, Kozhikode \\ ${ }^{3}$ Asso. Prof, Dept. of Prosthodontics, Govt. Dental College, Kozhikode \\ ${ }^{4}$ Junior Resident, Dept. of Prosthodontics, Govt. Dental College, Kozhikode \\ ${ }^{5}$ Senior Resident, Dept. of Prosthodontics, Govt. Dental College, Kozhikode
}

\begin{abstract}
It has been well documented that anterior ridge defects present in a patient are challenging to treat (especially aesthetically).To treat such defects when edentulous anterior portion of maxillary ridge has both inadequate height and width, the conventional options of fixed partial dentures or implant supported fixed partial dentures are not enough. In cases where replacement of teeth along with the supporting structures is necessary for aesthetics Andrew's Bridge is an option. The Andrew's bridge tends to be the best treatment plan for cleft palate patients with congenital or acquired ridge defect. This is designed to meet the requirements for esthetics, comfort,phonetics,oral hygiene and stress distribution to abutments and soft tissues.
\end{abstract}

Keywords: Andrew's bridge, anterior ridge defect, cleft palate, fixed-removable partial denture

\section{Introduction}

Restoration of missing teeth and lost alveolar contour presents a challenging task for the dentist in patients who have suffered from traumatic injury, congenital defects or other deformities which results in loss of anterior dentition along with the soft and hard tissue. In such cases, patient often desires a fixed prosthesis. But in cases of ridge defects, a tooth supported or implant supported fixed prostheses has several limitations: unaesthetic long pontics, lack of proper lip support, need of soft and hard tissue augmentation and difficulty in maintenance ${ }^{1}$. Siebert identified three basic ridge deformities: buccolingual loss of tissues (Class I) apicocoronal loss of tissues (Class II) and combination of a buccolingual and apicocoronal loss of tissues (Class III) ${ }^{2,3}$. Missing hard and soft tissues in cases of ridge defects can be treated in number of ways; one of the non-surgical treatment alternatives was introduced by Dr. James Andrews (Louisiana), as fixed removable Andrews Bridge System (Institute of Cosmetic Dentistry, Amite, La). This system is best indicated for cleft palate patients with congenital or acquired defects; its design meets the requirements of esthetics, comfort, phonetics, and hygiene maintenance along with favorable stress distribution between the abutments and residual soft tissues ${ }^{4}$. The design consists of two porcelain-fused-to-metal (PFM) crowns that fit over the abutments and both are connected by a bar that runs over the ridge. This assembly is permanently cemented to the prepared abutment, while the missing teeth are replaced by removable pontics with a metal sleeve tract embodied within an acrylic removable partial denture ${ }^{5}$.

\section{Case report}

A 17 year old female patient reported to the Department of Prosthodontics, Govenment Dental College, Kozhikode with a chief complaint of missing upper front teeth. Patient had a history of Grade III bilateral congenital facial cleft and had undergone surgery for the same followed by maxillary labial sulcus creation and columellar lengthening. Patient also underwent orthodontic treatment for the correction of bilateral posterior cross bite. On extra oral examination, a symmetrical face with morphologically altered nose and short columella were found. The facial profile was concave. Upper lip was short in both length and width (fig 1). Intra orally patient had retained 53 and 63, missing 12,22, 24 and 25, two palatally erupted supernumerary teeth in relation to 13 and 16 . A cleft in relation to 21 to 63 , mesially inclined 11 and 21 , buccally placed 12 and, the premaxilla was shifted to the right side. There was caries on 16 and 14. Radiographs showed root canal treated 11 and 21. Full metal crown was given in 26 and 46. In maxillary arch only 16 and 63 had occlusal contact with their complimentary teeth. The periodontal status and oral hygiene was fair (fig 2, $3 \& 4$ ).

From the examination findings, she was planned for a full maxillary arch rehabilitation with Andrew's bridge for replacing anterior teeth. The abutments of the Andrew's bridge were 21 and 63 and they were splinted to 13,1211 and 13. This segment was given as a single unit. Before commencing prosthetic phase all the carious teeth were restored. 
Preliminary maxillary and mandibular impressions were made with irreversible hydrocolloid impression material and the stone casts were obtained. Facebow transfer was done to orient the maxillary cast to the hinge axis of the semi- adjustable articulator (fig5) After the maxillary cast was articulated, interocclusal records were made to relate the mandibular cast with the maxillary cast. Interocclusal records were made with the patient in retruded mandibular jaw relationship using bimanual palpation method and wax record. Diagnostic preparations were made on the stone cast, and diagnostic wax up was done for the maxillary teeth. The diagnostic wax up was then completed with a group function concept of occlusion as the existing intercuspation was unacceptable and had to be changed. The deciduous teeth and supernumerary teeth were retained to minimize the alveolar bone resorption. Tooth preparation was done in 16, 15, 14,13,12,11,21,63,13 and 16. Provisional restorations were fabricated with the help of diagnostic wax-up by using indirect technique. It was cemented with temporary zinc oxide eugenol cement.

The provisional restoration was maintained intraorally for 4 weeks before final impressions were made, allowing sufficient time for evaluation. The patient was satisfied with the esthetics and comfort. The final impressions were made with elastomeric impression material ( Aquasil LV light body and Aquasil- soft putty regular set Dentsply ). The master cast was obtained and wax pattern was fabricated in the mounted cast. The metal frame works was then obtained and try-in done in the patient's mouth followed by porcelain application. A pick up impression was made with elastomeric impression materials ( Aquasil LV light body and Aquasilsoft putty regular set Dentsply) for the fabrication of metal bar connecting 21 and 23 (Fig 6 \& 7). After completion of the whole restoration with the bar was finished and polished. The temporary fixed partial denture was removed and the fixed component of the Andrew's System was temporarily cemented over the prepared teeth. Then with the crowns in position, along with the bar, an elastomeric impression was made and a stone cast was poured. Later, the missing teeth were arranged in the wax rim and trial was done, which was replaced with pink colored heat cure acrylic resin with a clip placed in the palatal aspect to attach this RPD over the bar attachment (fig 8\&9). After evaluating the occlusal interference and subsequent adjustments the final finished prosthesis was cemented using temporary cement (freegenol temporary pack GC). The patient was trained to remove and insert the RPD fabricated over the fixed component of Andrew's Bridge and proper oral hygiene (including interdental brush) instructions were given to the patient. The patient was reviewed after two weeks and that the patient was reported that she comfortable and happy with the final outcome and had pleasing aesthetics and phonetics. Necessary adjustments were done and the fixed part of the Andrew's bridge was permanently cemented (Lining \& Luting Cement Gold Label GC) ( fig 10 \&11). She was instructed to attend periodic recalls and checkups for the maintenance of the prosthesis and the components.

\section{Discussion}

During treatment planning, case selection is the important part of the treatment as there was severe bone loss. The esthetic replacement of anterior teeth is a challenge, especially in the maxillary arch. This situation can be further complicated by the presence of a ridge deformity. These anatomic defects may seriously compromise the esthetics of the final restoration. All the treatment choices were explained to the patient; after the patients' final decision, the Andrew's bridge was the treatment of choice.

The absolute Indications of Andrew's bridge are excessive residual ridge defect, ridge defects/ jaw defects either due to trauma and/or surgical ablation and cleft palate patients with congenital or acquired defects. Relative Indications are often fixed partial denture failure with badly damaged, cracked or weakened teeth either by fillings or disproportionate retainers and it has been used in patients with periodontal problems too. The advantages of Andrew's bridge are it includes all the advantages of fixed and removable partial dentures with better aesthetics, hygiene along with better adaptability and phonetics, comfortable and economical for patients. There is no plate as in RPD,so no soft tissue impingement and the surrounding structures and the system acts as stress breaker while transmitting unwanted leverage forces ${ }^{67}$.

The Andrew's System is usually of two types based on the area of bar attachment-

a) Pontic supported.

b) Bone Anchored or Implant supported Andrew's Bar System.

Compared to the conventional RPD, Andrew's bridge is more stable as it is totally tooth borne. The occlusal forces are also directed to the long axis of the supporting teeth. The frictional fit of walls of bar and sleeve provides retention. In Andrew's bridge system no soft- tissue impingement occurs. So further ridge resorption can be avoided. The curved bar ensures that the lifting and depressing forces are equally shared by all sections. The RPD design also involves placement of clasps in aesthetic zone which is not desirable for a young female patient. ${ }^{8,9,10}$

In contrast to the conventional FPD, the pontic teeth are arranged during the esthetic try-in appointment. Interdental papilla can be replaced without black triangle. The flange of the pontic assembly is contoured to 
improve the comfort, esthetics and phonetics and to resist the possible torque during function. Moreover, the pontic assembly can be removed to facilitate hygiene procedures and may be relined if any the ridge resorption occurs. In this way a replacement or pontic section can cover a minimum of soft tissue, afford a means of replacement of teeth with optimum esthetic arrangement, compensates for soft tissue defects and could be removed by the patient for easy day-to-day hygiene. ${ }^{11,12,13}$

Recently, spark-erosion technique has been introduced in dentistry. It is made of a primary cast joining the implant and removable metal superstructure upon which the replacement teeth are processed. However the inherent problems associated with the fabrication of spark-erosion overdenture may limit availability of the technology. They require high laboratory precision, are bulky and tend to wear out. ${ }^{6,10,14,15}$

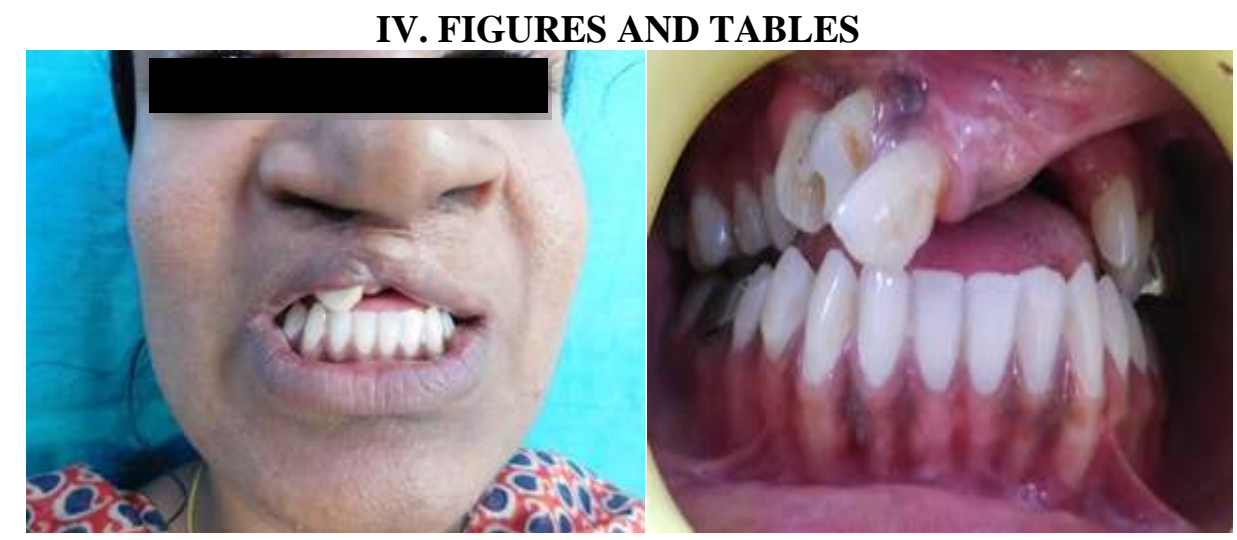

figure $1 \& 2$ : pre-treatment views

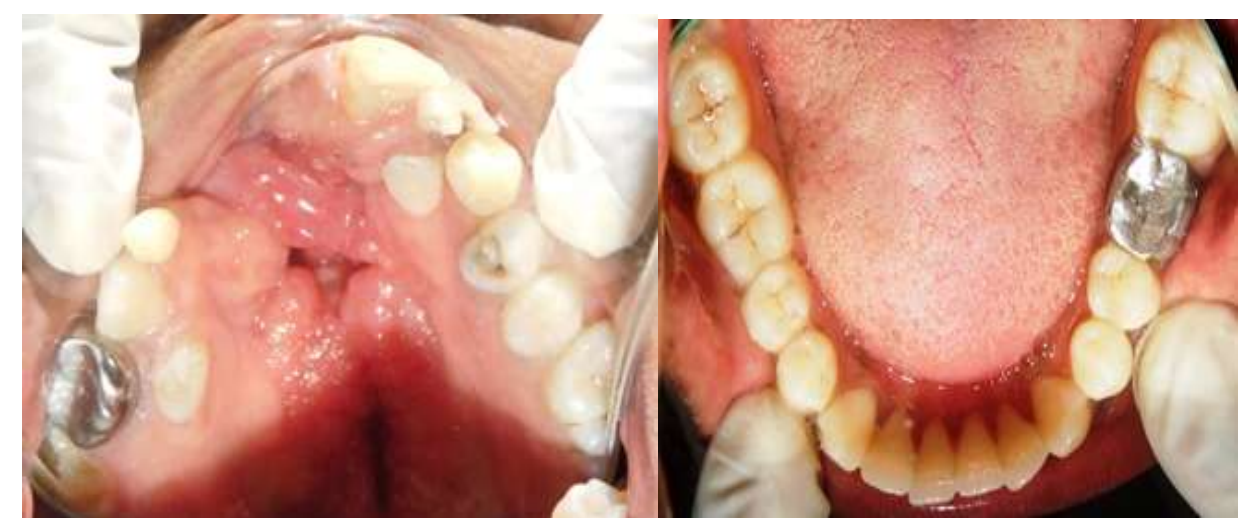

figure 3\&4: Pre- treatment occlusal views

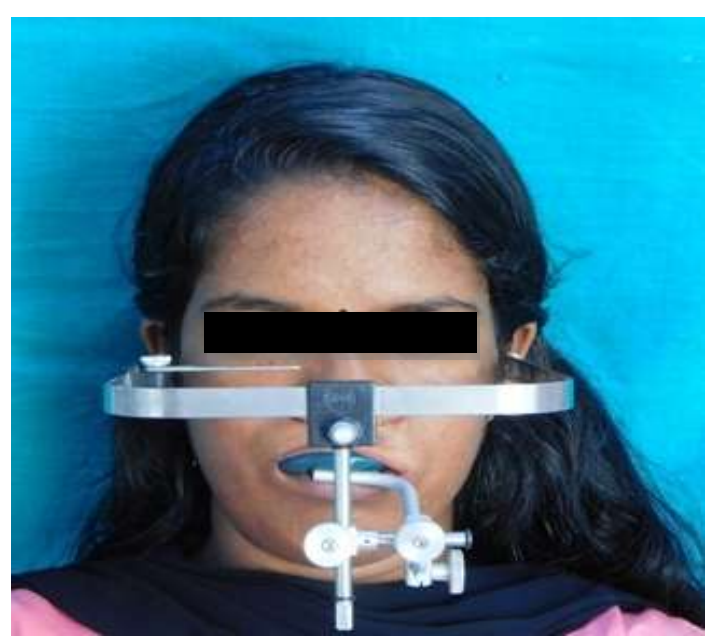

Figure 5: face bow transfer 

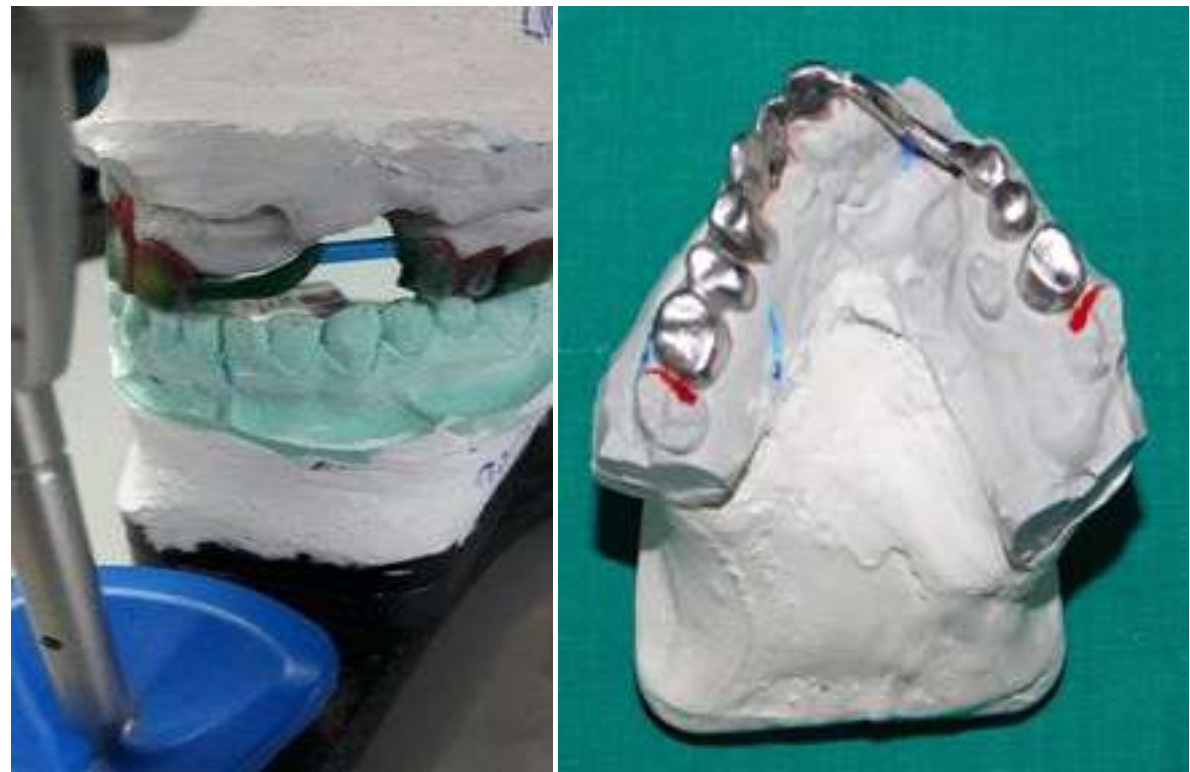

Figure 6: wax pattern of the frame work Figure 7: metal framework

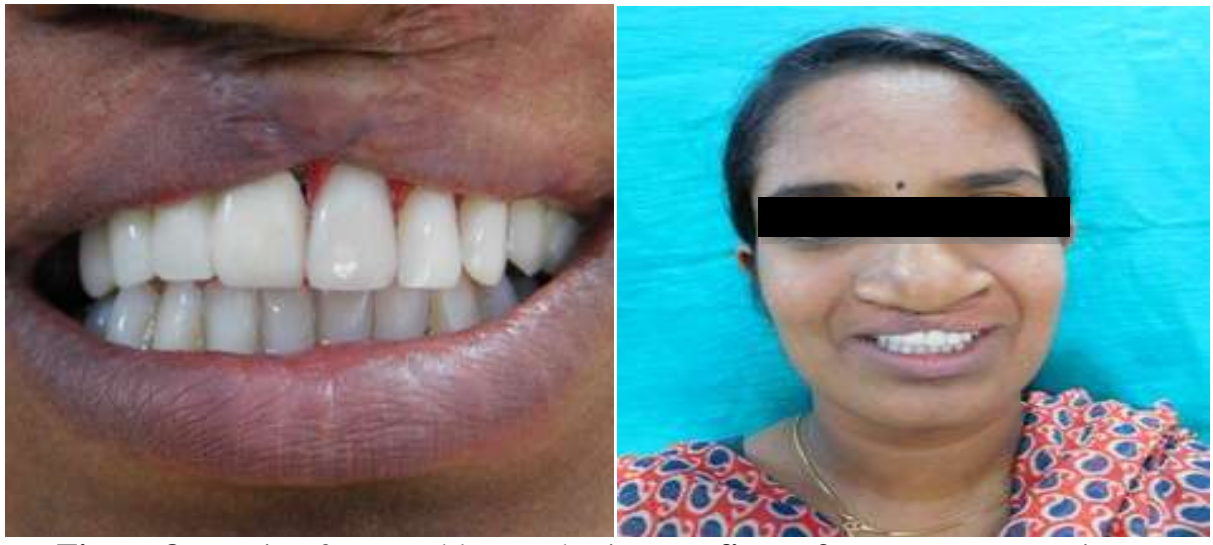

Figure 8: Try-in of removable prosthesis

figure 9: Post-treatment view

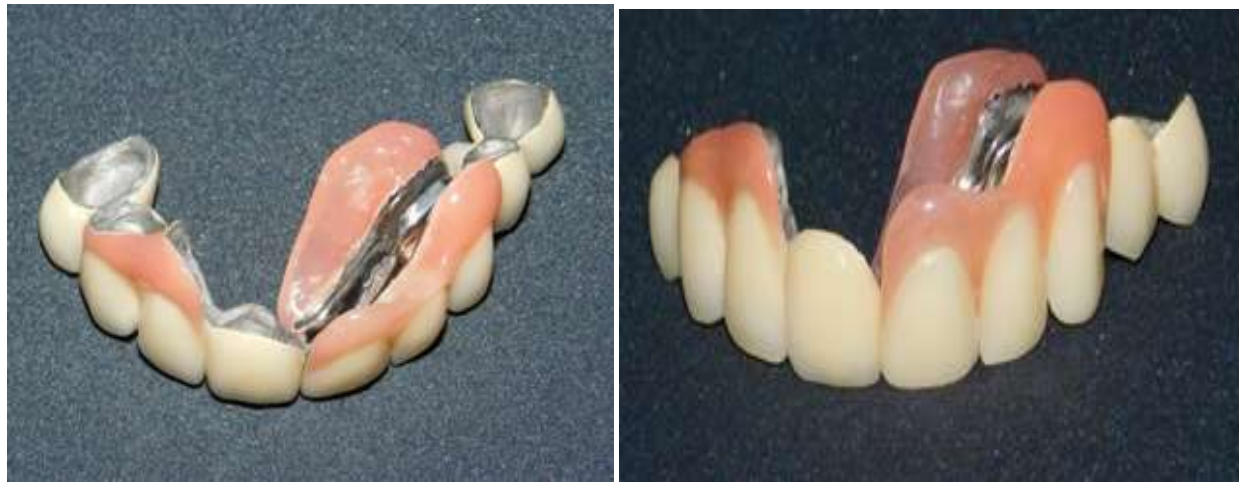

Figure 10\& 11 Andrew’s fixed-removable partial denture

\section{CONCLUSION}

Andrews Bridge system is a fixed-removable prosthesis that is indicated in patients with few missing teeth and large localized ridge defects. This functionally fixed prosthesis successfully replaces the missing teeth along with complete closure of the defect, restores speech and esthetics. The surgical grafting procedures still remain the best treatment options for the cases with unesthetic ridge defects. However, considering the treatment cost, invasiveness and treatment period in surgical procedures, the fixed removable system provides a rapid and economical substitute for achieving the desired treatment goals in cases with severe ridge defects 


\section{References}

[1]. Dr Priyanka Gubrellay et al, Andrews Bridge System - A literature Review International Journal Of Research In Dentistry 2014;4(3):59-62.

[2]. Pragati Kaurani, Rupandeep Kaur Samra, Mayank Kaurani, Narendra Padiyar Prosthodontic Rehabilitation Of A Case With An Anterior Ridge Defect Using Andrews Bridge Indian Journal of Dental Sciences. 2013;(2):100-3.

[3]. Seibert JS. Reconstruction of deformed, partially edentulous ridges, using full thickness onlay grafts. Part I. Technique and wound healing. Compend Contin Educ Dent. 1983;4(5):437-53

[4]. Vaswani P, sanyal P, kore A, prajapati A. Prosthodontic Rehabilitation of Anterior Bony Defect with Fixed Removable Bridge System: - A Claspless Approach. J Oral Bio. 2016; 3(1): 4

[5]. Kolaganti PK, Joshi S, Shalini BN, Kolaganti S, Jesudass G. Achieving Esthetics with Andrew's Bridge. Int J Prosthodont Restor Dent 2014;4(4):127-130.

[6]. Rai R, Menaga V, Kr G. A Prosthodontic Management of Severely Resorbed Anterior Ridge Defect - A Case Report. 2014;8(9):257.

[7]. Ahmad M, Balakrishnan D An Evidence Based Restoration of Esthetically Challenged Maxillary Anterior Arch with Andrews Bridge System - A Case Report with 5 Years of Follow Up. Dentistry (2016) 6: 357.

[8]. Shankar R. A Fixed Removable Partial Denture Treatment For A Sever Ridge Defect - International Journal of Dental Case Reports : 2016;1(2)

[9]. Walid MS Bone anchored Andrew's bar system a prosthetic alternative. Cairo Dent J (1995) 11: 11-15

[10]. Bhapkar P, Botre A, Menon P, Gubrellay P Andrew's bridge system: an esthetic option. J Dent Allied Sci 4: (2015) 36-40.

[11]. Rao Y, Yadav P, D'Souza M, Singh J, Jain A Bar and sleeve attachment: A report of two cases. J Clin Diagn Res (2013) 7: 30963098.

[12]. Muthuvignesh J, Bhuminanthan S, Egammai S, Donapati NR Improving facial esthetics with Andrew's bridge: a clinical report. Indian J Multidiscip Dent (2013) 4:884-887.

[13]. Kaurani P, Samra RK, Kaurani M, Padiyar N Prosthodontic rehabilitation of a case with an anterior ridge defect using Andrew's bridge. Indian J Dent Sci (2013) 2: 100-103.

[14]. Sadig WM. Bone anchored Andrews bar system.A prosthodontic alternative. Cairo Dent J.1995; 11(1): 11-5.

[15]. Salinas TJ, Finger IM, Thaler JJ 2nd, Clark RS. Spark-erosion implant supported overdentures: clinical and laboratory techniques. Implant Dent. 1992;1(4):246-51 